\title{
iPS cells strike a cord
}

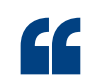

cord blood

banks ...

could become

an almost

unlimited

source of

clinically useful

stem cells.
Induced pluripotent stem (iPS) cells

— somatic and adult stem cells that are reprogrammed to a pluripotent state by the transient expression of various transcription factors - offer great promise for future regenerative therapies. Two studies, published in Cell Stem Cell, now report the successful generation of iPS cells from human umbilical cord blood. From a public perspective, cord blood is advantageous because of its ready availability, being routinely collected for public and commercial blood banks.

The production of iPS cell lines for clinical use faces two major issues. First, most iPS cells are generated from skin fibroblasts or other cell types that might require surgical intervention for harvesting. Second, cells isolated from adults could have accumulated mitochondrial and nuclear DNA mutations, which increase the risk of cellular anomalies and cancer. Cells from newborn cord blood allow both of these limitations to be overcome as they can be readily collected without any risk for the donor and are expected to carry minimal somatic mutations.
Ulrich Martin and colleagues produced iPS cells from human cord blood endothelial cells (CBECs). CBECs were transduced with lentiviral vectors expressing OCT4 (also known as POU5F1), SRY box-containing factor 2 (SOX2), NANOG and LIN28. When cells were cultured under human embryonic stem (ES) cell conditions, iPS cell colonies appeared with an efficiency as high as that previously reported for the reprogramming of fibroblasts and keratinocytes. The OCT4 promoter was demethylated in these cells, which confirms that they have an epigenetic status similar to that of ES cells. Cord blood-derived iPS cells were able to differentiate into all three germ layers in vitro. Furthermore, they could differentiate into cardiomyocytes similar to those in ventricular human heart tissue and to those obtained from human ES cells.

In another study, Juan Carlos Izpisua Belmonte and co-workers generated, with comparable efficiency, iPS cells from human cord blood stem cells. This reprogramming was achieved by expressing as few as two transcription factors - OCT4 and SOX2 - using retroviral vectors. OCT4 and SOX2 alone failed to reprogramme keratinocytes and fibroblasts, showing that cord blood stem cells have a higher capacity to acquire a pluripotent state. Importantly, the authors successfully obtained iPS cells from cord blood that had been cryopreserved for over 5 years, showing that this storage method, used in banked cord blood units, does not affect reprogramming.

These two studies open the possibility that cord blood banks, accessible worldwide, could become an almost unlimited source of clinically useful stem cells.

Kim Baumann

ORIGINAL RESEARCH PAPERS Giorgetti, A. et al. Generation of induced pluripotent stem cells from human cord blood using OCT4 and SOX2. Cell Stem Cell 5, 353-357 (2009)|Haase, A. et al. Generation of induced pluripotent stem cells from human cord blood. Cell Stem Cell $\mathbf{5}$, 434-441 (2009) FURTHER READING Nishikawa, S. et al. The promise of human induced pluripotent stem cells for research and therapy. Nature Rev. Mol. Cell Biol. 9, 725-729 (2008) 